\title{
Total Monte Carlo acceleration for the PETALE experimental programme in the CROCUS reactor
}

\author{
Axel Laureau ${ }^{1, *}$, Vincent Lamirand ${ }^{1,2}$, Dimitri Rochman ${ }^{2}$, and Andreas Pautz ${ }^{1,3}$ \\ ${ }^{1}$ Laboratory for Reactor Physics and Systems behaviour (LRS), Ecole Polytechnique Fédérale de Lausanne (EPFL), CH-1015 Lau- \\ sanne, Switzerland \\ ${ }^{2}$ Laboratory for Reactor Physics and Thermal Hydraulics (LRT), Paul Scherrer Institut (PSI), CH-5232 Villigen, Switzerland \\ ${ }^{3}$ Nuclear Energy and Safety Research Division (NES), Paul Scherrer Institut (PSI), CH-5232 Villigen, Switzerland
}

\begin{abstract}
The Bayesian Monte Carlo technics requires individual evaluations of random cross section files based on a Total Monte Carlo propagation. This article discusses the use of a Correlated Sampling acceleration applied to TMC calculations for experiments where a brute force technics is too expensive. An efficient estimation of the reaction rate uncertainties in small dosimeters is obtained, together with the inter-dosimeter correlation associated to the cross section uncertainties.
\end{abstract}

\section{Introduction}

The Bayesian Monte Carlo (BMC) is one of the assimilation technics used to provide feedback from critical reactors to the nuclear cross sections themselves. This technics is working together with a Total Monte Calo (TMC) uncertainty propagation able to provide observables of interest $\left(k_{\text {eff }}\right.$, activity, ...) calculations associated to a set of random cross sections such as displayed in Fig. 4 for the ${ }^{56} \mathrm{Fe}$. The comparison of measurements and calculations provides a $\chi^{2}$ value to each random cross section and then a weight is calculated: $\exp -\chi^{2} / 2$.
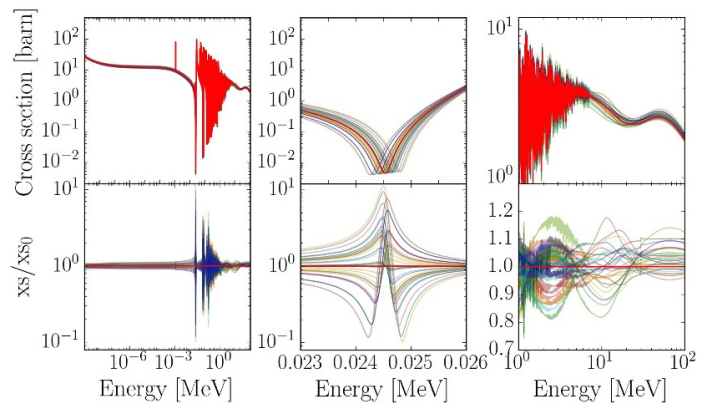

Figure 1. ${ }^{56} \mathrm{Fe}$ random Cross Section from the TENDL library

A very large number of random cross sections can be required in order to apply the BMC assimilation. This approach is already used [1] on fast critical systems with a large prior uncertainty and a short calculation time. If the evaluation of the random cross sections requires a large computation time, this step represents a limitation for the

\footnotetext{
*e-mail: axel.laureau@epfl.ch
}

applicability of the method. For the PETALE experimental program at EPFL, the observables are the reaction rates in small dosimeters located inside a metallic reflector of a water reactor that requires Monte Carlo transport calculations to be correctly estimated. The estimation of the dosimeter activities for a single random cross section can require days of calculation.

For this reason, as presented in this article, an acceleration of the TMC uncertainty propagation has been developed, based on a Correlated Sampling (CS) technics as detailed in Sec. 2. Two application cases are used to compare the developed method with reference and legacy approaches (Sec. 3): the HMI-001 benchmark and the PETALE setup itself. Finally, an approach able to quantify the correlation between the dosimeters for the nuclear data uncertainty is proposed in Sec. 4.

\section{TMC acceleration using the Correlated Sampling technics}

The correlated sampling [2] technics is a numerical method for Monte Carlo particle transport that allows to maintain the representativeness of two systems using a single particle track. For that, at each sampled event, the probability to do this event is calculated for the reference and the modified systems. For example, the system can be 'modified' according to dimensions, concentration, temperature or cross sections, the latter being the one of interest in this study. Then with a "modified weight" associated to the neutron transport, and then by multiplying this weight by the ratio of the two probabilities and finally by correcting all the scores using this weight, all the scores are representative of the two systems. A detailed presentation of this implementation in the Serpent2 [3] code is available in [4] for thermal feedback estimations. For 
example, for a neutron distance sampling $d$ with a total macroscopic cross section of $\Sigma_{t}$ in the reference system and $\Sigma_{t}^{\prime}$ in the modified system, the 'modified weight' multiplication term is: $\frac{\Sigma_{t}^{\prime} \exp \Sigma_{t} d}{\Sigma_{t} \exp \Sigma_{t}^{\prime} d}$. Note that this weight has to be propagated to the next generations of neutrons in order to take into account that the fission source is also modified.

In this study, the calculations are performed using the TENDL library. The first set of cross sections is considered arbitrarily as the "reference system". All the other random cross sections are considered as "modified systems". For each transported neutron, an array of modified weights corresponds to each modified system. An array of arrays of the neutron father's weight is also generated to take into account the fission source modification induced by the modification of the random cross sections.

The sampling distance and the type of interaction are considered here in the modified weight calculation. An unbiased estimation of the score modification due to these phenomena is expected even if a large cross section modification implies a slower convergence. A possible bias can be observed regarding the scattering anisotropy since the associated ratio of probabilities is not taken into account in this first implementation.

Considering an iron material definition with a perturbation of the ${ }^{56} \mathrm{Fe}$, and assuming that the Fe56.\#\#\#\#c element exists in the xsdata file where \#\#\#\# are the "0000, $0001,0002, \ldots$ ". random cross section files, the modified Serpent 2 input file using the correlated sampling on 256 libraries is given in Fig. 2

$\begin{array}{cccc}\text { mat mat_iron autopert } 0 & 256 & -7.874 & \\ \text { Fe54.03C } & 0.05845 & \text { Fe56.0000C } & 0.91754 \\ \text { Fe57.03C } & 0.02119 & \text { Fe58.03C } & 0.00282\end{array}$

Figure 2. Iron material definition with 256 different ACE files

During the Serpent2 initialisation phase, an optional "autopert" key has been added in the material definition. When declared, all the isotopes defined with ".0000c" are then automatically duplicated for the correlated sampling with the labels ".0000c", ".0001c", ".0002c"...

\section{Validation}

The overall objective of the current work being the integral experimental program PETALE (see section 3.3) associated to the stainless steel at high neutron energy, the HMI-001 benchmark has first been selected for its high sensitivity to the ${ }^{56} \mathrm{Fe}$ uncertainty. It consists of a highly enriched uranium/iron metal core reflected by a stainlesssteel reflector. In a second step, the approach is also validated on the reaction rates of dosimeters located in the PETALE experimental setup.

\subsection{Comparison of uncertainty propagation approaches}

Three technics are considered here to compute the TMC uncertainty propagation.
The "TMC-Ref" is the classic Total Monte Carlo uncertainty propagation technics. It requires $\mathrm{n}$ independent calculations for $\mathrm{n}$ random cross section files. This technics provides a reference distribution of the propagated uncertainty.

The "TMC-CS" is the new TMC uncertainty propagation using the correlated sampling technics. Up to 128 random cross section files are propagated in a single calculation. Multiple calculations are then combined altogether using the same "reference system".

Finally the "TMC-sensi" is based on sensitivity calculations. The sensitivity array is estimated using Serpent2, and represents the $k_{\text {eff }}$ variation due to a $1 \%$ perturbation of the cross sections in a given energy range. Associated to the actual cross section relative variations between the cross section files Fe56.\#\#\#\#c and Fe56.0000c, the uncertainty is propagated for each random cross section file.

\subsection{HMI-001 benchmark}

\subsubsection{Classic TENDL library}

The effective multiplication factor distribution according to the random cross sections is presented in Fig. 3 We can

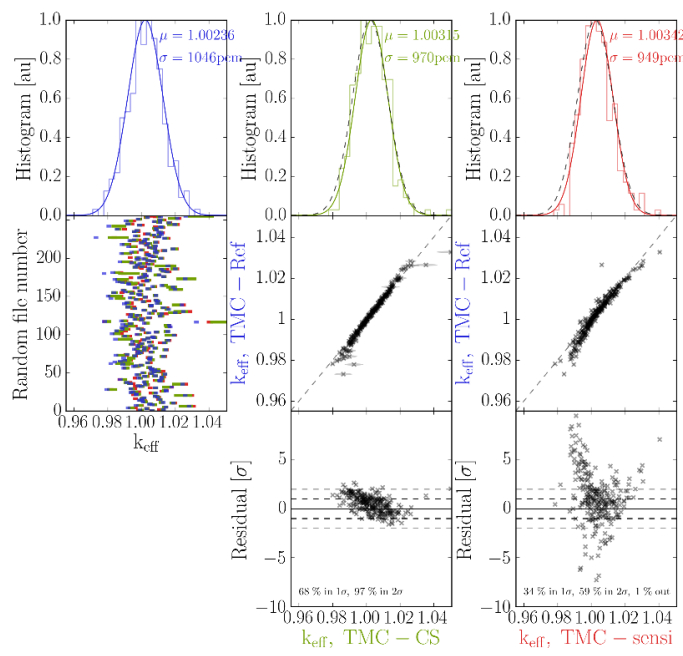

Figure 3. $k_{\text {eff }}$ distribution associated to the ${ }^{56} \mathrm{Fe}$ uncertainty. The plots on the first line respectively present the results of the TMCRef, TMC-CS and TMC-sensi approaches. The second line corresponds to the $k_{\text {eff }}$ values and the agreement between the three approaches for each value. The third line presents the associated residuals.

see that the impact of the ${ }^{56} \mathrm{Fe}$ cross section on the $k_{\text {eff }}$ is significant, around $1000 \mathrm{pcm}$ of uncertainty. We also observe that the three uncertainty propagation technics provide a standard deviation with a very good agreement.

Concerning the difference between the $k_{\text {eff }}$ calculation for a same random cross section but using different methods (second line Fig 3), the global trend is linear. Using the TMC-CS, all the $k_{\text {eff }}$ calculations are compatible with 
the TMC-Ref results. The residuals are good even if a slightly non-linear effect seems to appear, possibly due to the angular effect that is not taken into account. A nonlinearity appears on the sensitivity approach when the reactivity variation (and then the cross section variation) is too large. The global trend is not linear but looks like a coma curve, resulting in larger residuals even if the global agreement is good.

\subsubsection{Extended TENDL library}

The classic TENDL library performs a sampling of the nuclear parameters and compares the generated cross sections to the EXFOR database in order to obtain random cross sections. In the extended TENDL library used here, the sampling is performed on the nuclear models themselves in order to have a larger cross section dispersion and thus to better check the validity of the methods.

Figure 4 shows the dispersion of the cross sections (left) using different sets of nuclear models, each model being declined with the sampling of the model parameters. We can see that two packs of cross sections are visible at high energy for the inelastic and capture cross sections. Fig. 4-right presents the uncertainty propagation using these cross sections. A larger non-linearity appears with two distinct coma lines, each line being associated to a pack of cross sections with a discontinuity between the two.
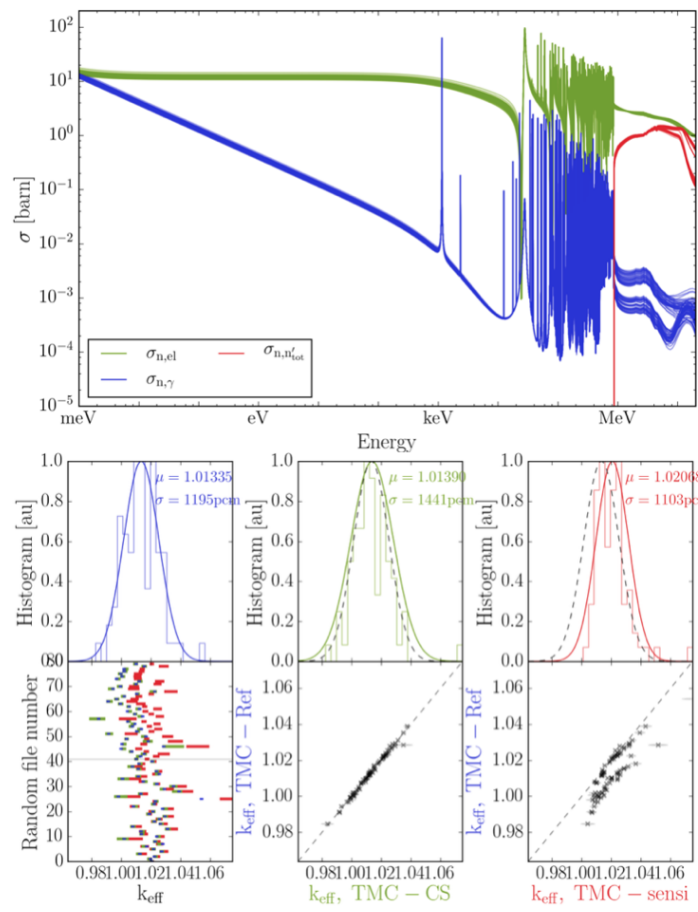

Figure 4. Extended TENDL library cross sections and corresponding uncertainty propagation (see legend of Fig. 3

\subsection{Dosimetry in the PETALE setup of the CROCUS reactor}

The HMI-001 benchmark illustrates the good capability of the TMC-CS technics to predict reactivity variations associated to the random cross sections. This section aims to validate the method for reaction rate estimation in dosimeters.

Figure 5 presents the PETALE setup in the CROCUS reactor. A full description of the experiment is available

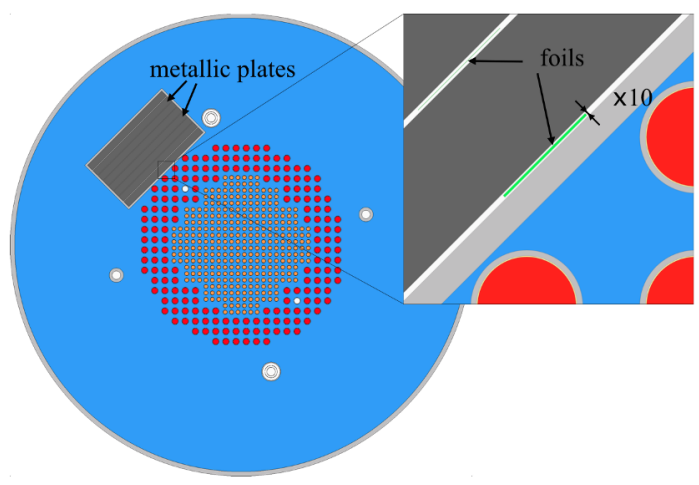

Figure 5. Serpent 2 CROCUS geometry with the PETALE experimental setup

in [5] and the description of the experimental program is available in [6]. The PETALE setup is composed of 8 metallic plates (in gray) interleaved with nine dosimeters (in green). In this paper the metallic reflector is composed of pure iron, and the dosimeters are made of indium in this section.

During the calculations, the reaction rate score is declined using different modified weights that correspond to the different sets of random cross sections. The corresponding reaction rates are presented in Fig. 6. We can see that the reaction rates as a function of the dosimeter number (top) have a very different pattern between the capture (left) and the inelastic (right) reactions. This is due to the different characteristic energies of the neutron doing the interaction: at high energy for the threshold inelastic cross section and at thermal energy for the capture reaction. The impact of the random cross sections is visible through the curve dispersions, and the relative variation is presented on the second line. We can see that this dispersion reaches a maximal value of around $15 \%$. A very good agreement is obtained between the TMC-Ref (dashed lines) and the TMC-CS (plain lines) calculations. We also observe that the statistical uncertainty on the reaction rate variation is reduced with the TMC-CS. With the classic TMC-Ref approach, a difference between two independent calculations with their independent statistical uncertainties $\sigma_{1}$ and $\sigma_{2}$ leads to a total uncertainty of $\sqrt{\sigma_{1}^{2}+\sigma_{2}^{2}}$, while the correlated sampling technics directly provides the estimation of the score difference using the same neutron tracks and then this results in a reduced statistical uncertainty. 

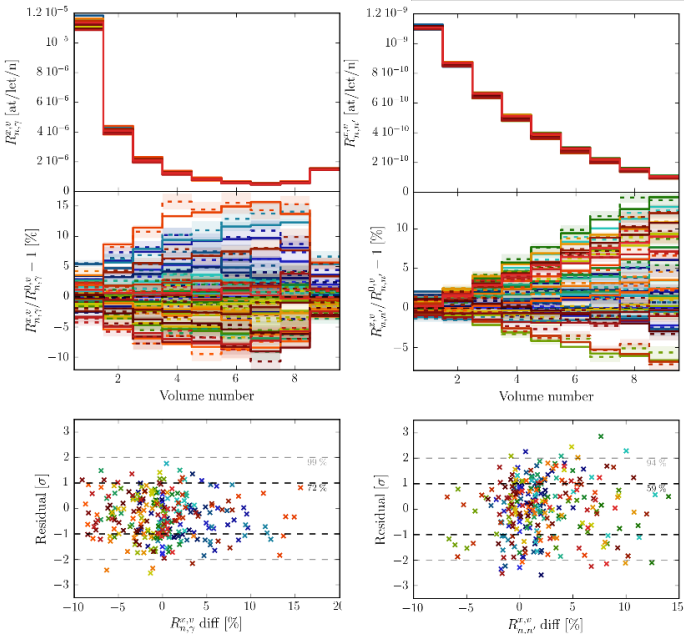

Figure 6. Reaction rate distributions (top) for the capture (left) and inelastic (right) reactions; relative variations with one color per random cross section of the classic TENDL library (second line) with the TMC-CS in plain lines and the TMC-Ref in dashed lines; and residuals (bottom)

\section{Dosimeter correlations}

The TMC-CS is used to estimate the reaction rate values in dosimeters for multiple random cross sections with a single calculation. Multiple dosimeters can be used, by putting the dosimeters side by side or using distinct calculations. The first solution is considered in this section.

Figure 7 shows the reaction rate dispersion for two threshold reactions: the rhodium and the indium inelastic reactions. One can see that the ordering between the
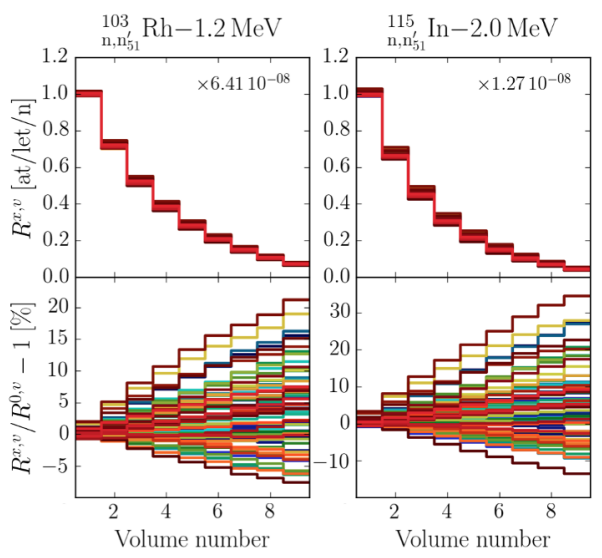

Figure 7. Inelastic reaction rate distributions (top) for the rhodium (left) and indium (right) elements and the relative variation between the random cross sections (bottom)

random cross section curves is very similar. For example, in volume 8 , even if the relative variation is larger for the rhodium $(20 \%)$ compared to indium $(30 \%)$, the ordering is the same: brown curve for the maximal, then the yellow curve, the blue, the brown, the green one... This means that, if we assume that the measurement has no experimental uncertainty and that there is no nuisance parameters, the two dosimeters will provide the same information. For example if we also assume for a simplification purpose that one of the TENDL random cross sections (corresponding for example to \#42 associated to the blue color in the figure) is very close to the real one, then the indium measured value will be the blue curve, and the BMC will provide a large weight for all the curves with a similar value (the yellow, blue, brown ones). And then since the ordering is the same with the rhodium dosimeter, the selected curves will be identical. In the opposite situation, a different uncorrelated distribution would provide a distinct information and allows to discriminate between the remaining cross section files.

For this reason we can use the correlation matrix of this reaction rate dispersion in order to discriminate between independent dosimeters and quantify the level of correlation. Figure 8 presents this correlation matrix for a set of dosimeters. We can see that as expected the rhodium and

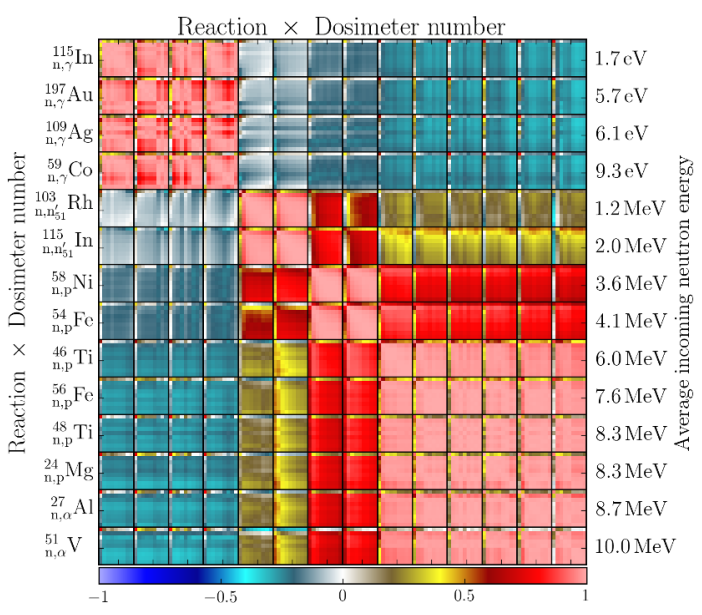

Figure 8. Correlation matrix of the reaction rate dispersion after the TMC-CS uncertainty propagation with different dosimeters ordered by the lethargy average incoming neutron energy. Each sub-matrix in the diagonal corresponds to one element with the correlation of the different positions (nine distinct dosimeters), and the sub-matrix off-diagonal corresponds to the inter-element correlations.

the indium results are very correlated. We also obtain a correlation coefficient for all the different dosimeters. This correlation will be useful for the BMC assimilation in order to avoid assimilating several times the same information. This is a useful element regarding the experimental program as discussed in [6]. 


\section{Conclusions}

The correlated sampling technics is used to propagate random cross sections through a single Monte Carlo calculation. This propagation has been validated for iron cross sections by comparison with a reference Total Monte Carlo approach. Two gains are obtained with this technics: a strong reduction of the number of Monte Carlo calculations; and thanks to the estimation of the score difference using a single neutron path, the variance on the difference is reduced. This method appears to be very useful for integral experiments requiring long calculations with a small statistical uncertainty, such as reaction rate estimations in a reflector.

\section{References}

[1] Rochman, D. A., Bauge, E., Vasiliev, A., Ferroukhi, H. and Perret, G., EPJ Nuclear Sciences \& Technolo- gies, 4, 7 (2018).

[2] Rief, H., Annals of nuclear energy, 11, 9, 455-476 (1984).

[3] Leppänen, J., Pusa, M., Viitanen, T., Valtavirta, V. and Kaltiaisenaho, T., Annals of Nuclear Energy, 82, 142150 (2015).

[4] Laureau, A., Buiron, L. and Fontaine, B., EPJ Nuclear Sciences \& Technologies, 3, 16 (2017).

[5] Lamirand, V., Perret, G., Radman, S., Siefman, D. J., Hursin, M., Frajtag, P., Gruel, A, Leconte, P., Blaise, P. and Pautz, A., ISRD16 International Symposium on Reactor Dosimetry (2017).

[6] Lamirand, V., Laureau, A, Rochman, D., Perret, G., Gruel, A, Leconte, P., Blaise, P. and Pautz, A., International Workshop On Nuclear Data Evaluation for Reactor applications, (2018). 